\title{
Sublimation Driven Ionization for use in Mass Spectrometry: Mechanistic Implications
}

Charles N. McEwen, ${ }^{1,2 *}$ Ellen D. Inutan, ${ }^{2,3}$ Abigail Moreno-Pedraza, ${ }^{4}$ I-Chung Lu, ${ }^{5}$ Khoa Hoang, ${ }^{2}$ Milan Pophristic, ${ }^{2}$ SarahTrimpin ${ }^{2,4 *}$

${ }^{1}$ University of the Sciences, Chemistry, Philadelphia, PA 19104

${ }^{2}$ MSTM, LLC, Newark, DE 19711, USA,

${ }^{3}$ Mindanao State University Iligan Institute of Technology, Iligan City 9200 , Philippines

${ }^{4}$ Department of Chemistry, Wayne State University, Detroit, MI 48202, USA

${ }^{5}$ National Chung Hsing University, Taichung City, Taiwan 402

Corresponding author: c.mcewen@usciences.edu 


\section{Ion Yield of vMAl}

The relative ion-to-neutral ratio of MALDI ion a Waters SYNAPT G2 was calculated by the measured total ion abundance and the total number of desorbed neutrals. To calibrate the ion detection efficiency in this instrument, the ion abundance was multiplied by a parameter $5 \times 10^{-2}$ to fit the absolute ion-to-neutral ratio of MALDI from the previous report.1,2 The solid squares show acceptable fitting results in Figure 1, indicating that we can use the same fitting parameter to estimate the absolute ion abundance of MAI. Figure 2 shows the chromatogram of the pure MAI matrix (3-NBN). The total number of acquired ions was calculated by integrating ion intensity and then multiplying the fitting parameter $\left(5 \times 10^{-2}\right)$. The number of desorbed 3-NBN molecules from the sample surface is $2 \times 10^{18}$; therefore, the ion-toneutral ratio is around $1.3 \times 10^{-13}$. Compared to the ion-to-neutral ratio of MALDI matrices $\left(10^{-9}-10^{-6}\right)$, the ion yield of the MAI matrix is much lower. The same procedure was employed to evaluate the ion yield of analyte in MAI. The total ion abundance of doubly charged bradykinin was estimated considering the subtraction

of matrix related ions. The ion yield of analyte (bradykinin) was $1.1 \times 10^{-8}$, which is again lower than it is in MALDI $\left(10^{-4}\right) .^{3}$ This evaluation underestimates the ion yield of MAl, because ionization occurs during the ca. 2 minutes the sample is under vacuum and being loaded into the region of ion transmission. The tail of the ionization event is shown in Figure 2. However, this order of magnitude still has value for reference. Even though the ion yield of MAI is low, the ionization induced by sublimation allows us to collect more ion signals at one time compared to the spot-tospot acquisition in MALDI.

\section{Experimental}

The ionization efficiency of MAI was briefly measured by Waters SYNAPT G2 (QIMS-TOF) mass spectrometer. Since the ion yields of MALDI matrices were reported previously, ${ }^{1,2}$ the detection efficiency of ions can be determined by calibrating the measurement at the same laser fluence. MALDI-grade materials, CHCA and 2,5 DHB, purchased from Sigma Aldrich (St. Louis, MO, USA) were used in this study. The stock solutions of matrix were prepared by dissolving the corresponding compounds separately in a $75 \%$ acetonitrile aqueous solution $(0.01 \mathrm{M})$. The sample was prepared by dry-droplet method on the sample plate $(0.5 \mu \mathrm{L})$ shown in Figure $1(\mathrm{a})$ and (c). We use scanning mode to irradiate sample spot-to-spot until a quarter of the MADLI sample was eliminated (Figure 1(b) and (d)). The total ion abundance of MALDI was calculated by integrating overall ion intensity in the chromatogram at various laser fluence, respectively (Figure 2). The number of desorbed neutrals (1.25 $\mathrm{nmol}$ ) was estimated from the number of molecules in the irradiated region (a quarter of the whole sample). 
The total ion abundance of MAI was measured by the same instrument and identical setting to MALDI experiments, but without the use of laser. For evaluating the different compounds for the MAI matrix, 3-nitrobenzonitrile (3-NBN) matrix (5 mg) was dissolved in $75 \%$ acetonitrile aqueous solution $(50 \mu \mathrm{L})$. The analyte, Bradykinin (20 pmol) was mixed with matrix solution per $0.5 \mu \mathrm{l}$, and then co-crystalized on the sample plate by dry-droplet method.

\section{References}

1. I-Chung Lu, Chuping Lee, Yuan-Tseh Lee, and Chi-Kung Ni. Ionization Mechanism of Matrix-Assisted Laser Desorption/lonization. Annual Review of Analytical Chemistry, 8, 21-39 (2015)

2. I-Chung Lu, Kuan Yu Chu, Chih-Yuan Lin, Shang-Yun, Wu, Yuri A. Dyakov, Jien-Lian Chen, Angus Gray-Weale, Yuan Tseh Lee, Chi-Kung Ni. Ion-toNeutral Ratios and Thermal Proton Transfer in Matrix-Assisted Laser Desorption/Ionization. Journal of The American Society for Mass Spectrometry, 26(7), 1242-1251 (2015)

3. Kuan Yu Chu 1, Sheng Lee, Ming-Tsang Tsai, I-Chung Lu, Yuri A Dyakov, Yin Hung Lai, Yuan-Tseh Lee, Chi-Kung Ni. Thermal proton transfer reactions in ultraviolet matrix-assisted laser desorption/ionization. Journal of The American Society for Mass Spectrometry, 25(3), 310-8 (2014) 
Figures

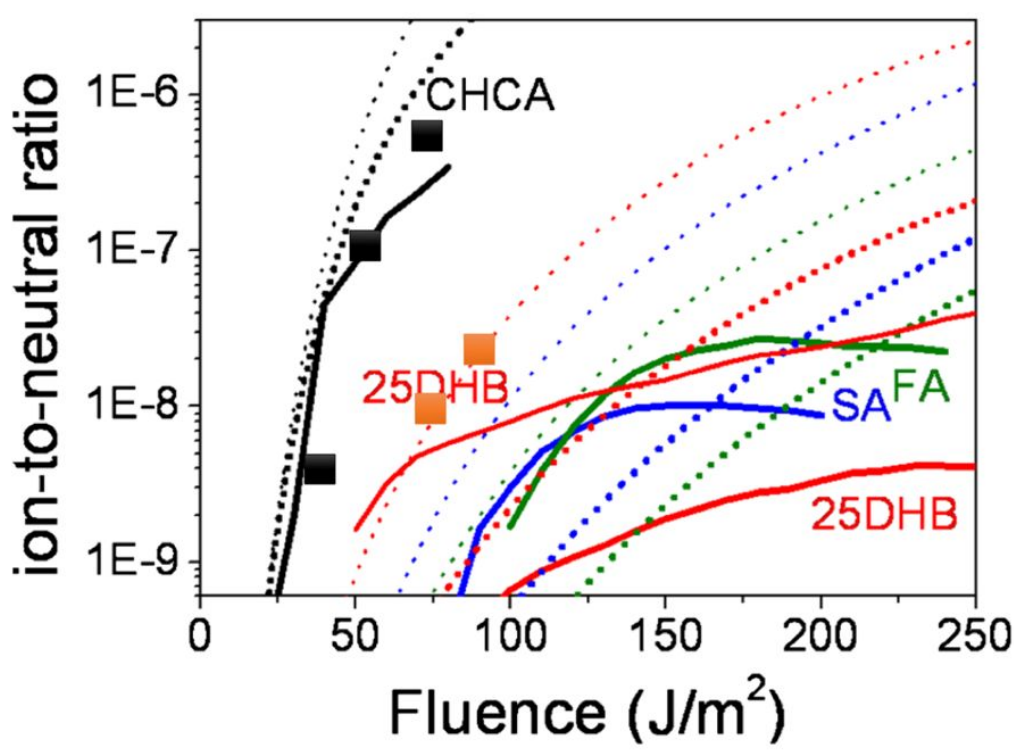

Figure S1: Ion to neutral ratio vs. Fluence obtained on a homebuilt MALDI-Tof. ${ }^{2}$

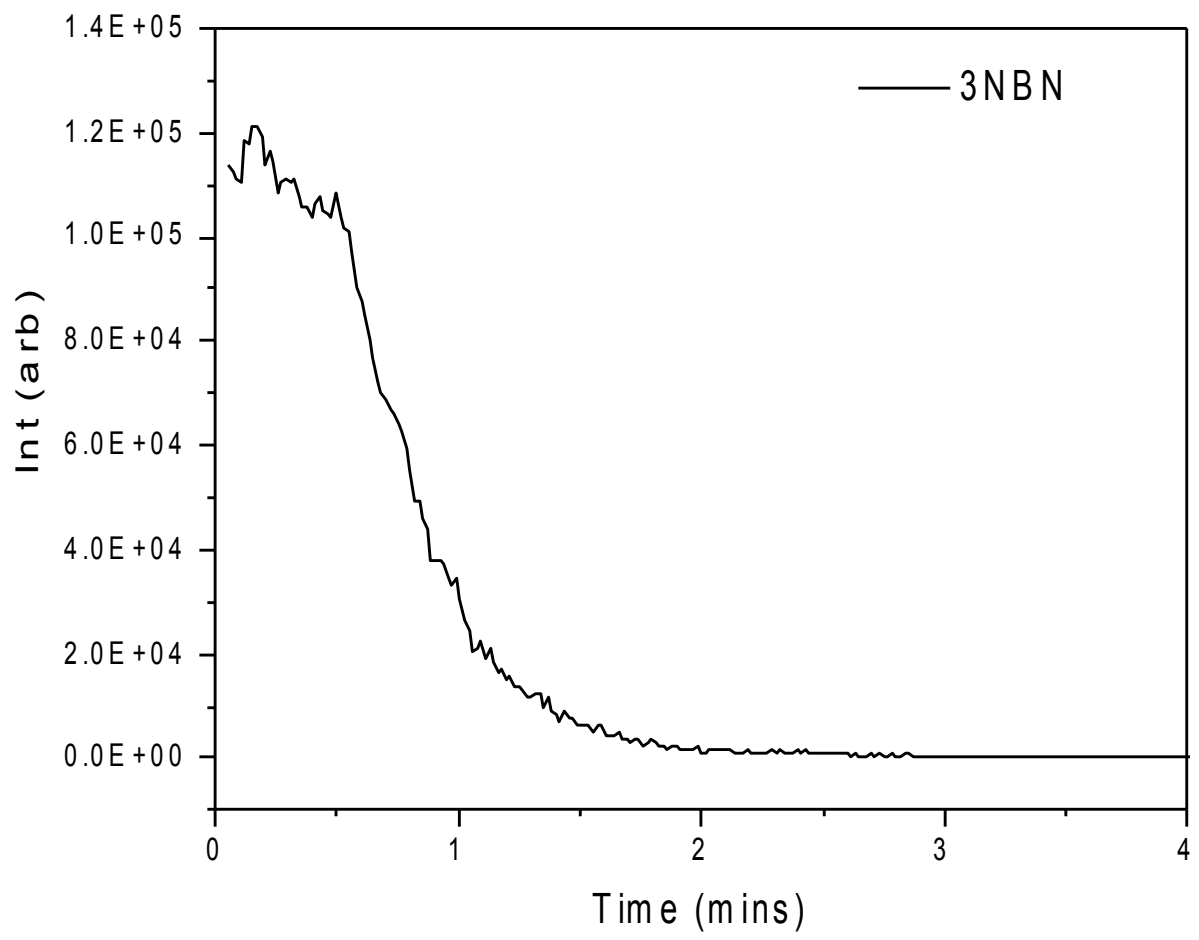

Figure S2: lon abundance for the 3-NBN matrix recorded vs. time on a Waters SNYPT G2 mass spectrometer. 


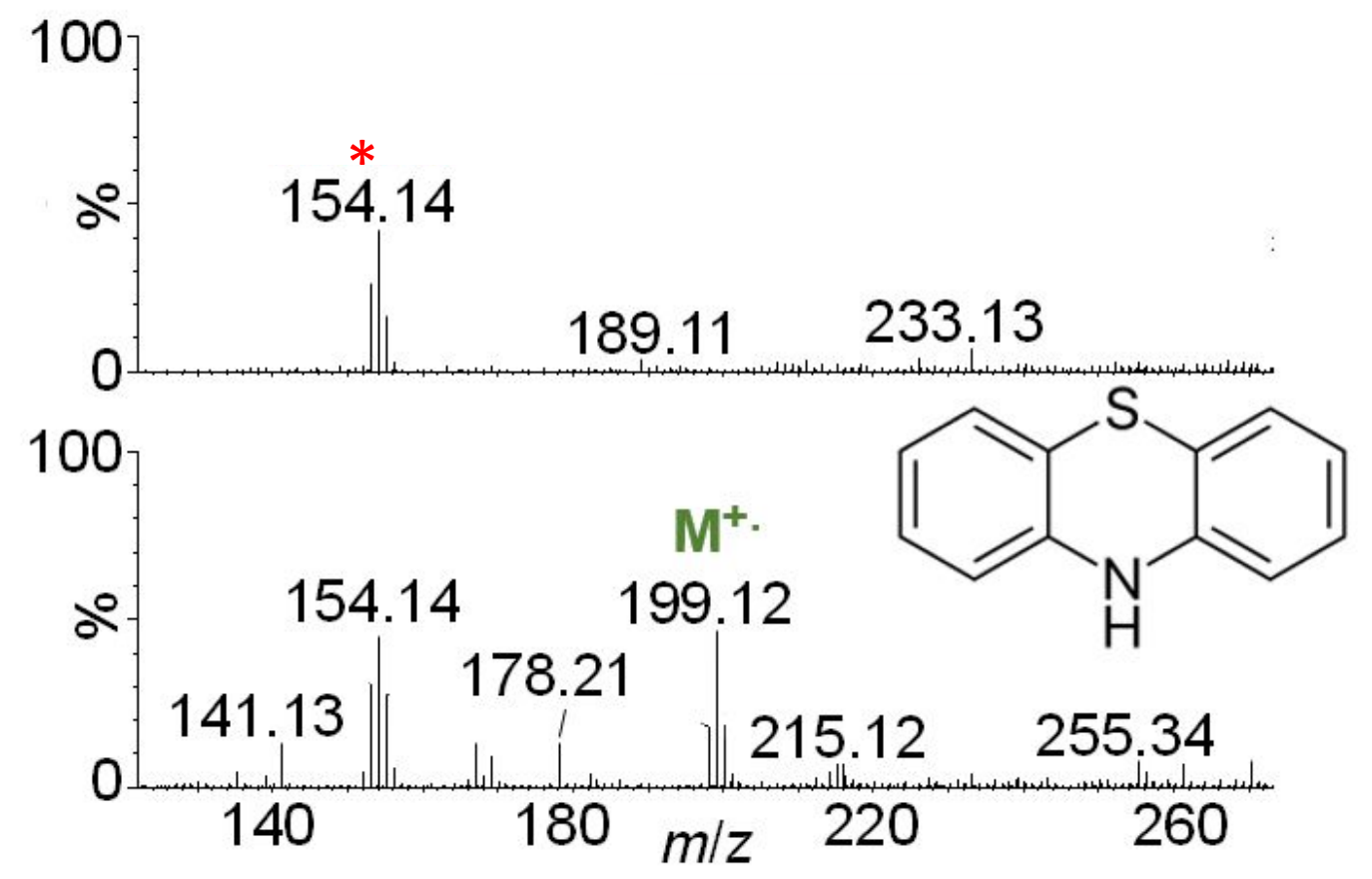

Figure S3: Mass spectra of (A) acenaphthene (MW 154) and (B) phenothiazine (MW 199) using acenaphthene as a vMAl matrix with sample insertion into a Waters SYNAPT G2S mass spectrometer using the probe method. The major ion observed for acenaphthene is its radical cation as is also the case for phenothiazine when using acenaphthene as matrix. 


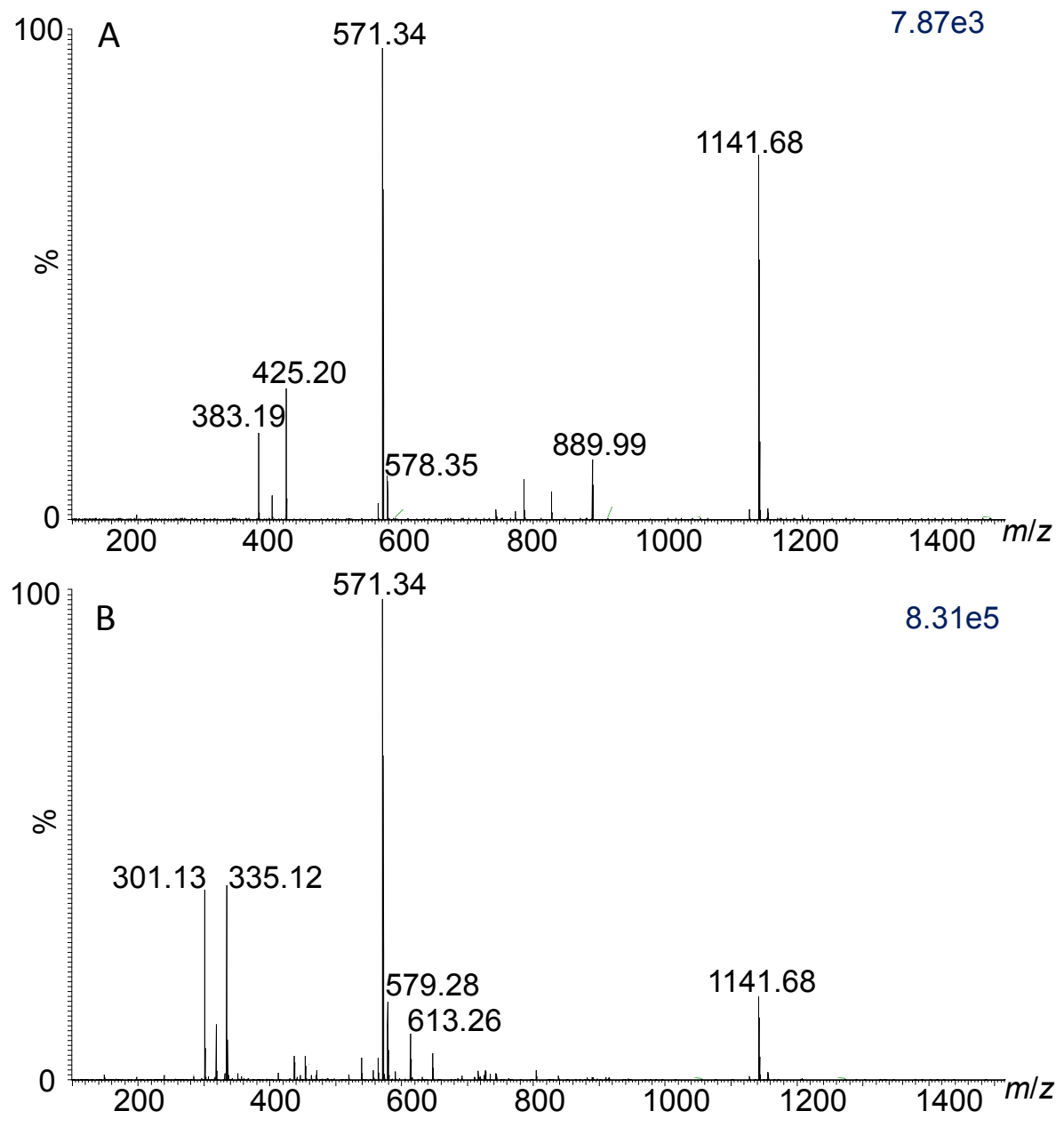

Figure S4: VMAI mass spectra of $500 \mathrm{fmol}$ of gramicidin S obtained using the MSTM prototype high throughput vMAI source on a Thermo Q-Exactive Focus and using 3NBN as matrix with A) no heat applied to the ion transfer tube lens, and B) the tube lens heated. The increase in ion abundance of the doubly charged ion at $\mathrm{m} / \mathrm{z} 571.34$ and singly charged ion at $\mathrm{m} / \mathrm{z} 1141.68$ of gramicidin $S$ is related to more rapid sublimation of the matrix in $\mathrm{B}$ and possibly better desolvation of charged matrix:analyte particles. 

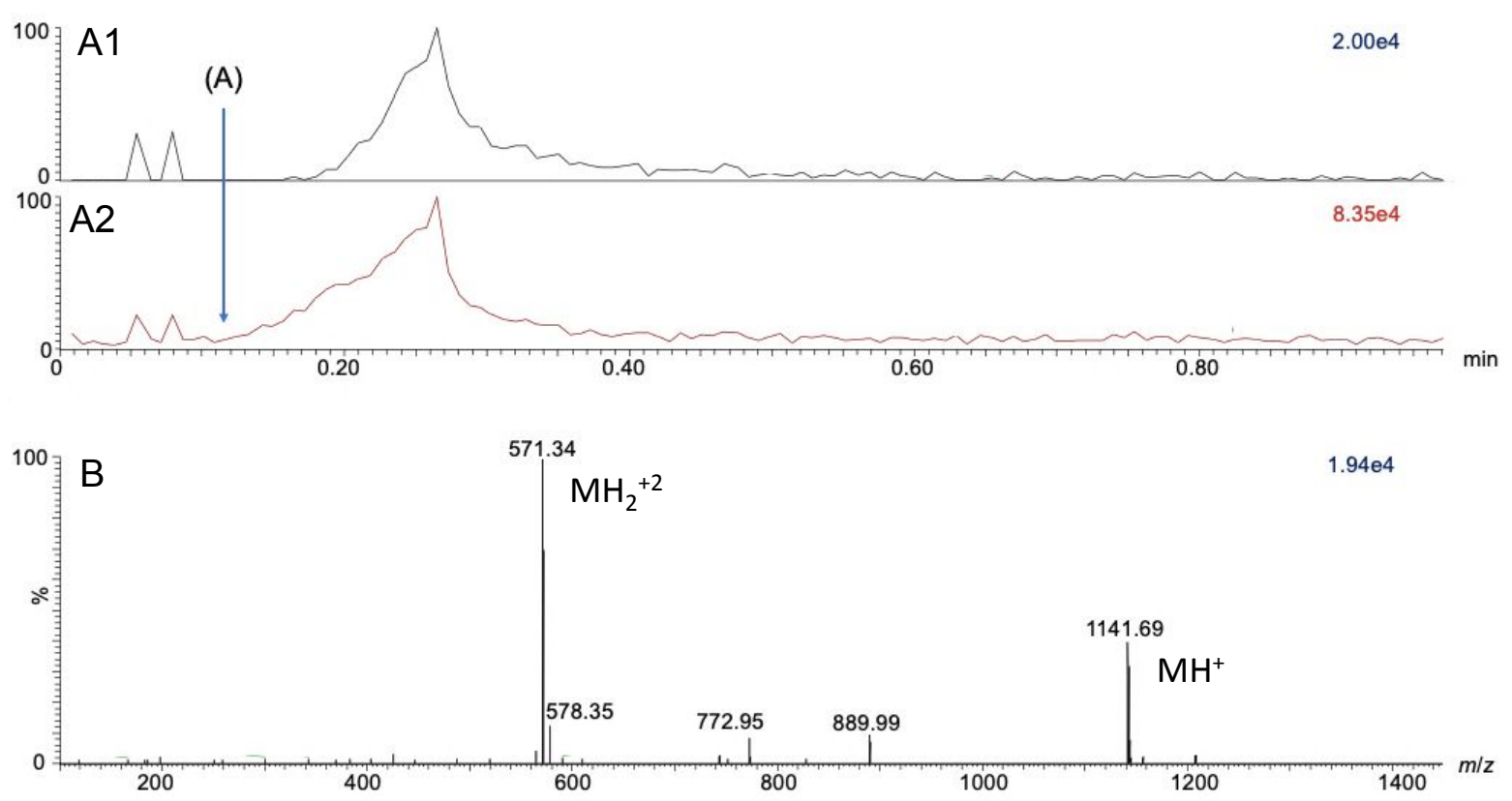

Figure S5: Total ion current (A2) and ion abundance of the doubly charged ion of gramicidin S ( $\mathrm{m} / \mathrm{z} 571)(\mathrm{A} 1)$ and summed mass spectrum (B) from application of 0.2 $\mu \mathrm{L}$ of 3-NBN matrix solution containing $500 \mathrm{fmol}_{\mu \mathrm{L}^{-1}}$ of gramicidin $S$ onto a glass slide, dried, and exposed to the vacuum of a Q-Exactive Focus mass spectrometer using the MS ${ }^{\mathrm{TM}}$ prototype high throughput vMAI source. (A) represents the time at which the sample was exposed to vacuum. 

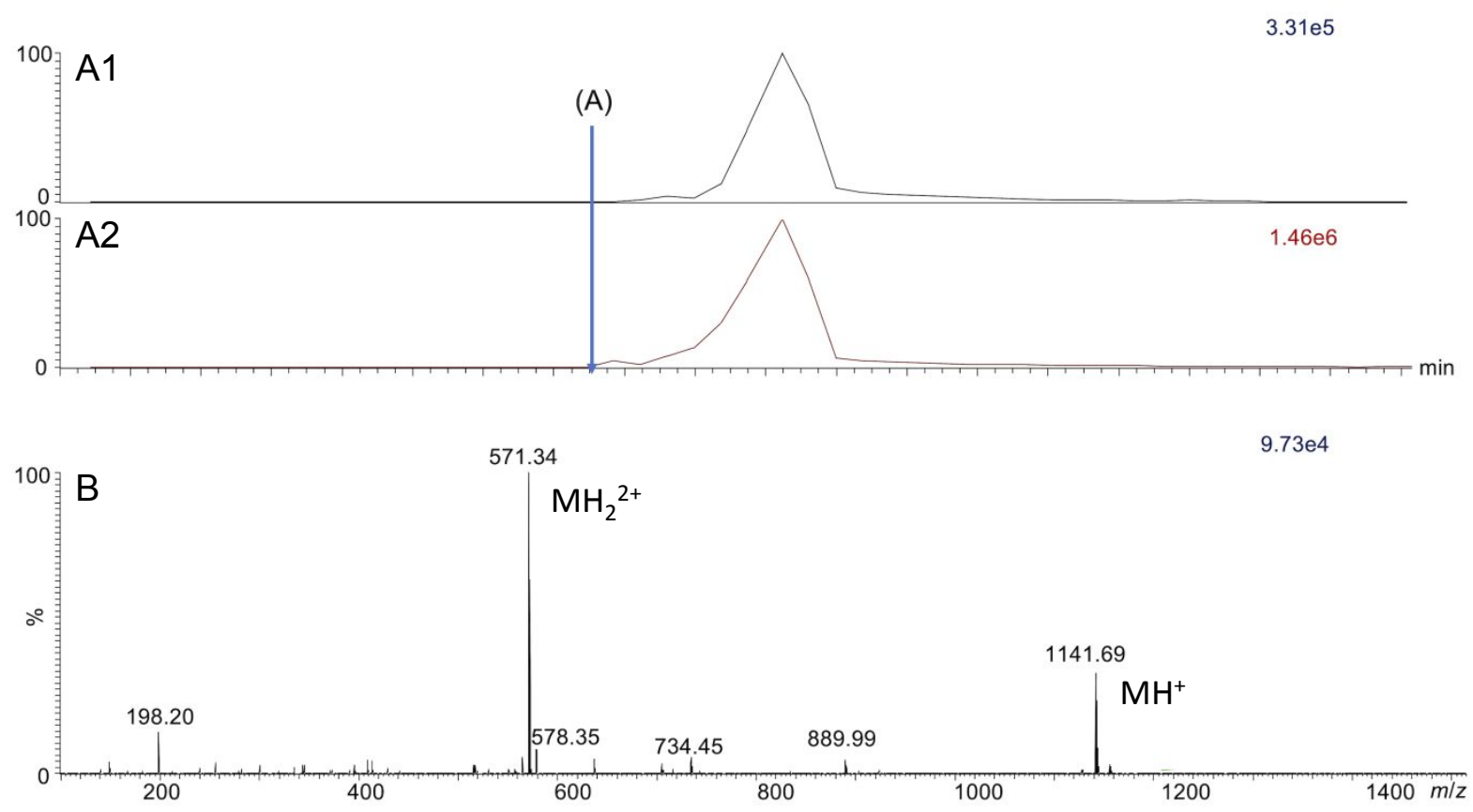

Figure S6: Total ion current (A2) and ion abundance of the doubly charged ion of gramicidin S ( $\mathrm{m} / \mathrm{z}$ 571) (A1) and summed mass spectrum (B) from application of 0.2 $\mu \mathrm{L}$ of a 1:0.15 w/w ratio binary 3-NBN:methyl 2-methyl-3-nitrobenzoate matrix solution

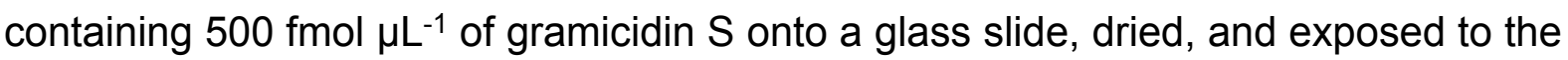
vacuum of a $\mathrm{Q}$-Exactive Focus mass spectrometer using the MSTM prototype high throughput vMAI source. (A) represents the time the sample was exposed to vacuum. 


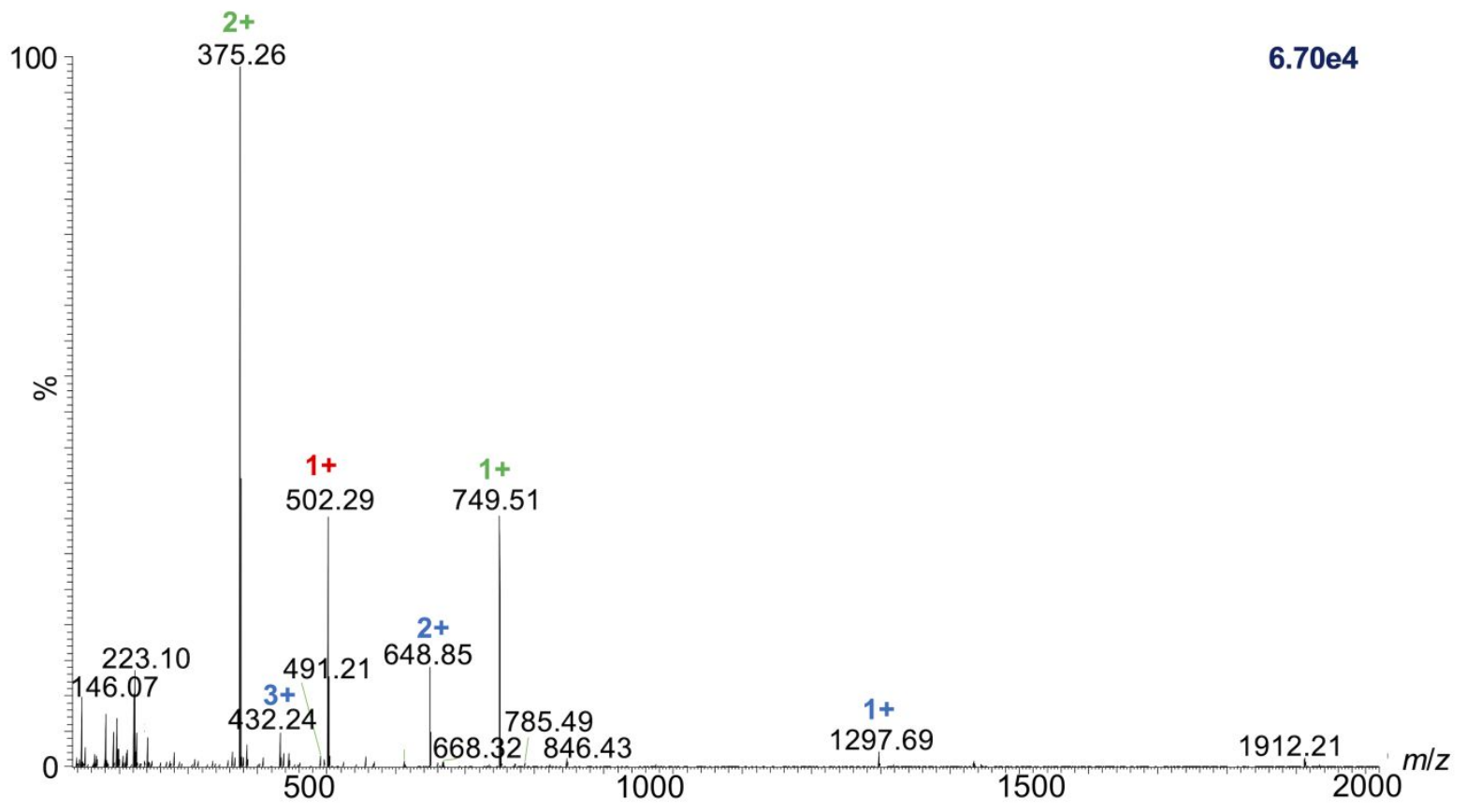

Figure S7: vMAI probe mass spectrum obtained on the Q-Exactive Focus using $100 \%$ DCB as matrix and analytes fexofenadine $(\mathrm{m} / \mathrm{z} 502)$, azithromycin $(\mathrm{m} / \mathrm{z} 375.2$ and 749.5, angiotensin I ( $\mathrm{m} / \mathrm{z} 432.2,648.8$, and 1297.9), and insulin $(\mathrm{m} / \mathrm{z}$ 1912.2). 


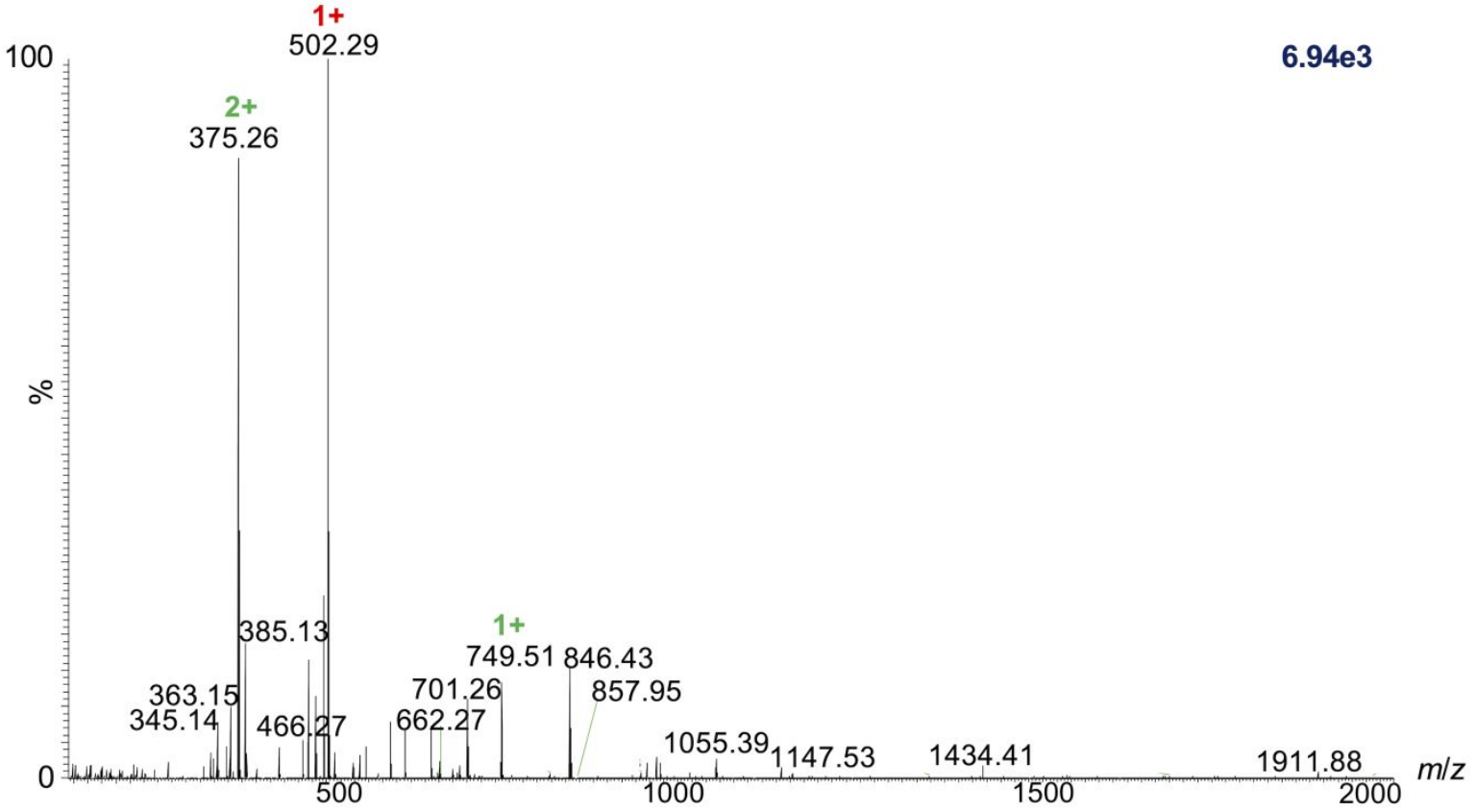

Figure S8: vMAI probe mass spectrum obtained on the Q-Exactive Focus using $100 \%$ DNB as matrix and analytes fexofenadine $(\mathrm{m} / \mathrm{z} 502)$, azithromycin $(\mathrm{m} / \mathrm{z} 375.2$ and 749.5, angiotensin I (not observed), and insulin ( $\mathrm{m} / \mathrm{z}$ 1147.4, 1434.4, and 1912.2). 


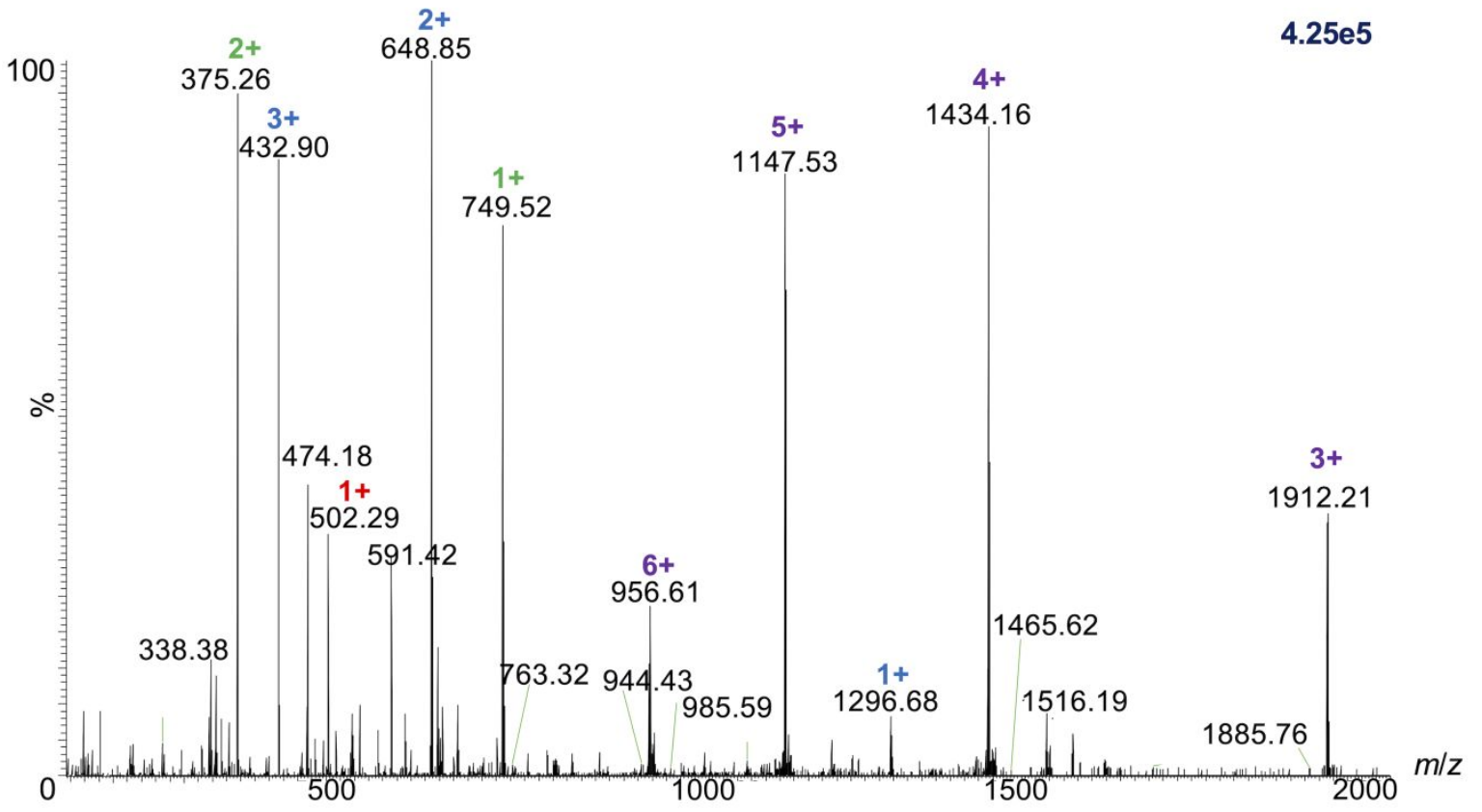

Figure S9: vMAI probe mass spectrum obtained on the Q-Exactive Focus using a 1:1 molar mixture of DCB and DNB as matrix and analytes fexofenadine $(\mathrm{m} / \mathrm{z} 502)$, azithromycin ( $\mathrm{m} / \mathrm{z} 375.2$ and 749.5, angiotensin I $(\mathrm{m} / \mathrm{z} 432.9,648.8$, and 1296.7), and insulin $(\mathrm{m} / \mathrm{z}$ 956.6, 1147.5, 1434.2, and 1912.2). 